\title{
EFFICACY OF DYNAMIC MUSCULAR STABILIZATION TECHNIQUES IN THE MANAGEMENT OF LUMBAR DISC DISORDERS
}

\author{
Journal website at; \\ http://mrtbjournal.org/index.php/njmr/issue/current/showToc
}

\begin{abstract}
SURAJ KUMAR ${ }^{1}$,VIJAI P.SHARMA', RAKESH SHUKLA ${ }^{2}$, RAVI DEV $^{3}$, ANOOPAGGARWAL

'Department of Physical Medicine and Rehabilitation, Chatrapati Sahuji Maharaj Medical University

${ }^{2}$ Department of Neurology, Chhatrapati Sahuji Maharaj Medical University, Lucknow- 226003, India.

${ }^{3}$ Department of Neurosurgery, Chhatrapati Sahuji Maharaj Medical University, Lucknow-226003, India. ${ }^{4}$ Department of Physiotherapy, Pt.DDU IPH, New Delhi-110002,India.anoop_bpt@yahoo.com
\end{abstract}

\author{
Correspondence to: \\ Suraj Kumar \\ E-mail :- surajdr2001@yahoo.com \\ Mobile no.: + 91-98890-92835
}

\begin{abstract}
Background: Lumbar disk disorders (LDD) are common in low back pain (LBP). Stabilization techniques are effective in the management of LDD. But their effectiveness in different disc levels is not well documented.
\end{abstract}

Objective: This study determines the efficacy of Dynamic muscular stabilization technique (DMST) in different disc involved in patients with LBP retrospectively.

Methods: Total 76 patients were categorized in 5 groups on the basis of number of discs involved. The identification of LDD was done by MRI. All subjects were treated with DMST, an active approach of stabilizing training. Pain was the primary outcome measure while physical ability (walking, stair climbing, stand ups), physical strength (back pressure change: $\mathrm{BPC}$, abdominal pressure changes: APC) and quality of life (QOL) were the secondary. Variables were assessed at baseline (day 0 ) and at the end of the follow up (day 180).

Results: Results showed that the pre treatment severity differed between the groups but their mean difference were found not significantly different ( $P>0.05)$. The DMST improved all the groups (disc involved) significantly $(\mathrm{P}<0: 01)$.

Conclusions: This study conclude that pain, walking, stair climbing and BPC improved maximally in the subject involving lesser number of discs whereas the quality of life improved more in the subjects with higher number of discs involved.

Key Words: Low Back pain, Physiotherapy, Rehabilitation, Segmental, Stabilization, Strengthening Exercises

\section{INTRODUCTION}

Lumbar disc disorder is (LDD) due to a change in the structure of the normal disc and are common in Low back pain (LBP). LDD comes as a result of aging and the degeneration of that occurs within the disk. As the disc continue to degenerate with continued stress on the spine, the inner nucleus pulposus may actually rupture out from the annulus called herniated disc. The fragments of the disc can then press on the nerve roots that are located just behind the disc space which causes pain, physical limitation and alter physical strength of the effected area. Based on the history and physical examination LBP is classified into nonspecific and specific. Non specific diagnosis of chronic LBP, to which lumbago can be referred are most common, with a prevalence of about $90 \%$ to $95 \%$ in the early phase of the chronic condition (Borenstein, 1996). Specific LBP are those, which have known cause such as infection, trauma, neurological disorder, cancer, fracture, inflammatory disorder and cauda equine syndrome etc.

To identify scrious pathology, all guidclines for management of LBP have recommended use of a diagnostic triage. There are many existing diagnostic tools for LBP such as $x$-ray, magnetic resonance imaging (MRI), 
computed tomography (CT) scan, nerve conduction tests and myelography etc, but most of them are not cost effective and conflicting. Study also showed that disc degeneration is seldom found in patient under 10 years old identified by MRI (Salo et al, 1995). However, at present no reliable and valid classification system exists for diagnosis and prognosis of LBP (Koes et al, 2006).

Due to injury, the deep stabilizing muscles of the lower back will remain impaired for 4-6 weeks until pain subsides. In LBP, impaired deep stabilizing muscles provide poor segmental stiffness, and thus predispose the back to reinjury and return to pain. Therapeutic exercise, as part of rehabilitation for patient with LBP, is one of the treatment modalities most commonly used by physiotherapists (Martin et al, 1986). In the management of such cases, the dynamic muscular stabilization techniques (DMST) were also found to be effective (Lucy et al, 2006). In DMST, adequate dynamic control of lumbar spine forces is achieved which reduces the repetitive injury to the structures of the spinal segments and related structures. Specific stabilizing exercises with cocontraction of deep abdominal (transversus abdominis) and lumbar multifidus muscles enhance the spinal segmental support and control (Richardson, 1995). Therapeutic exercise, as part of rehabilitation for patient with LBP, is one of the treatment modalities most commonly used by physiotherapists (Martin et al, 1986), physical exercises have also been proved to be effective in the management of LBP both in short term and long term (Hides et al, 2001). In our earlier studies, we have found DMST is more effective than Conventional treatment in the management of sub-acute and chronic LBP (Kumar et al, 2008a; Kumar et al, 2008b). Though stabilization techniques were found suitable LDD, but their effectiveness in different disc levels is not well document. We hypothesized that DMST may improve single or multiple disc involved equally.

\section{MATERIAISANDMETHODS Subject}

A total 98 LBP patients (age 20-30years) were seen at the Department of Physical Medicine and Rehabilitation, CSM Medical University, Lucknow. All patients were diagnosed clinically by a physician as having nonspecific, sub-acute or chronic low back pain (CLBP). Lumbo-sacral spine MRI of 76 patients showed disc degeneration, herniation, stenosis, hypertrophy, spondylosis or spondylolysthesis. All patients were divided into five groups on the basis of the number of disc(s) involved. For example, in figure 1 one disc is involved consist group 1 and in figure 2 two discs were involved is group 2 and similarly, 3, 4, and 5 (Figure not shown). These groups may include one or more MRI findings. Subjects were excluded from the study if they suffered from any known specific neurological disorder or muscular degenerative condition such as muscular dystrophy or if they had undergone any lumbar spine surgery, infection, vascular problem and leg pain/or low back pain for less than 6 weeks.
The present study had the approval of the Institutional Review Board and informed consent was obtained from all the participants.

Ethical approval was obtained from the Institutional Ethics Committee of CSM Medical University (reference letter no. 734/R-cell-07).

\section{Approach}

The outcome measure were severity of pain (Pain), physical ability (Walking, Stair climbing and stand-ups) physical strength (BPC and APC) and quality of life (QOL). All of these were assessed by same tester and same physiotherapist supervising the test procedure at base line (day 0 ) as well end of the treatment and follow-ups (day 180). Follow up was started after 20 days of regular exercises at OPD, and it ended after 6 months from the 0 day. During follow up, subjects had an appointment periodically with the investigator at 15 days interval for review of exercises. Test and retest of two groups were conducted in the same place at same environment and at the same time of the day. Before experimentation, all subjects were well taught about the measurement variables and their outcomes. The patients were also informed about the experimental risks, if any.

\section{Procedure}

All subjects were treated with DMST treatment by the same physiotherapist with the same intensity and capacity in all the patients on 20 regular days and followed up for 180 days. The duration of each individual treatment session was approximately 40 minutes per day. In DMST, muscles with direct attachment to lumbar spinal segment stabilize the joint's 'neutral zone' and prevent excessive deflection. Details of exercise, which were given in four stages, were presented elsewhere (Kumar et al, 2008a; Kumar et al, 2008b).

\section{Outcome variables}

The level of pain was assessed by visual analogue scale (VAS: $0-10 \mathrm{~cm}$ ) (Jensen et al, 2002) while functional ability (Walking, Stair climbing and Stand up) was assessed with Waddle functional evaluation test (Waddell, 1998). The Physical Strength were back pressure change (BPC) and abdominal pressure changes (APC) measured by Pressure measuring device and overall QoL by SF-36 questionnaire. The measuring details of variables in brief are summarized as follows:

Visual analogue scale (VAS) - This is a $10 \mathrm{~cm}$ calibrated line with 0 representing no pain and 10 representing worst pain. The subjects were asked to make a mark or point on the scale that best represents his/her intensity of pain experienced on the same day.

Walking - The distance walk up and down between marks 10 meter apart in 5 minute. The corridor was quiet and empty with non-slip surface or hard carpet. Patient can not use any walking aid but can use the walls for support or can sit down for a rest. Regular information about the 
time was given to the patient between walking.

Stair climbing - Climbing up and down of standard stairs with one handrail and opposite wall within easy reach were used. Stair climbing counts of a patient was taken as total steps ups and downs completed in one minute for example a patient can up stairs 10 steps and down 18 steps, the total counts are 28 .

Stand ups - The number of times the patient can stand up from a chair in 1 minute is his score. The chair was firm, upright with a backrest but no armrest. The seat height of chair was 45 centimeter. During stand up there was no support within reach so that patient cannot use any support.

Physical strength - The physical strength (BPC and APC) was measured by pressure measuring device (PMD). The measuring details of BPC and APC and of PMD were presented elsewhere (Kumar et al, 2008a). All the measurements were taken at baseline (day 0 ) and at the end of the follow-up (day 180).

$S F-36$ quality of life - It is a multipurpose, self administered, short form (SF) health survey with 36 questions which measures generic health status on general population. These questions consists physical functioning, role functioning, bodily pain, general health, vitality, social functioning, role functioning and mental health. Response choices are numbered from left to right, starting with 1 . The maximum scores obtained from 36 questions were 151 represents worst QoL whereas minimum score 36 represents the best.

\section{Statistical analysis}

Before analyzing the data, the homogeneity of variance testing between groups of all pre outcome measures were done separately by Bartlett's test and their significance of mean difference between groups were done by one way analysis of variance ANOVA) followed by Newman Keuls (Q test) post hoc test. After that the two periods (pre and post) and five groups were further compared together by two factor ANOVA followed by Newman Keuls (Q test) post hoc test. A two tailed (á=2) probability $(P)$ values less than $0.05(P<0.05)$ was considered to be statistically significant. Microsoft EXCEL (MS Office 97-2003) and GraphPad Prism (version 5) were used for the analysis.

\section{RESULTS}

\section{Distribution of Subjects}

There were 38 subjects in group $1(50.0 \%), 19(25.0 \%)$ in group $2,9(11.8 \%)$ in group 3 and $5(6.6 \%)$ each in group 4 and group 5 (Table 1 ).

\section{Outcome Measures \\ Homogeneity of variance}

The homogeneity of variance testing and significance of mean difference between groups were summarized in Table 2. Table 2 showed that the groups of pre outcome measures were homogeneous $(P>0.05)$ and their mean values were similar i.e. did not differed significantly $(P>0.05)$

\section{Effect of treatment}

The pre and post outcome measures data were summarized in Table 3 and also shown graphically by Fig 3. The ascending order of severity of pre outcome measures follows as

Pain : 2 disc $<1$ disc $<4$ disc $<3$ disc $<5$ disc

Walking : 1 disc $<2$ disc $<3$ disc $<4$ disc $<5$ disc Stair climbing: 1 disc $<2$ disc $<4$ disc $<3$ disc $<5$ disc Stand ups: 1 disc $<4$ disc $<2$ disc $<5$ disc $<3$ disc BPC : 2 disc $<1$ disc $<3$ disc $<4$ disc $<5$ disc

APC $: 4$ disc $<2$ disc $<1$ disc $<3$ disc $<5$ disc QoL $: 5$ disc $<4$ disc $<3$ disc $<1$ disc $<2$ disc

The above comparison showed that the severity differ among groups but their mean difference were found to be not significant $(P>0.05)$ except pain between 3 discs with, 1 disc and 2 discs and walking between 1 disc with 5 discs.

Similarly, comparing between periods (Fig 3) the improvement of DMST on outcome measures follows the same trend i.e. 1 disc improved the most, followed by 2 disc, 3 disc, 4 disc while 5 disc the least $(5$ disc $<4$ disc $<$ 3 disc $<2$ disc $<1$ disc). All groups improved significantly $(\mathrm{P}<0.01)$ except walking in 4 disc and 5 disc.

\section{DISCUSSION}

Table 1: Distribution of all subjects $(n=76)$ as per disc involved

\begin{tabular}{lll}
\hline $\begin{array}{l}\text { Number of disc } \\
\text { involved }\end{array}$ & $\begin{array}{l}\text { Subjects } \\
\text { (no) }\end{array}$ & $\begin{array}{l}\text { Subjects } \\
(\%)\end{array}$ \\
\hline 1 & 38 & 50.0 \\
2 & 19 & 25.0 \\
3 & 9 & 11.8 \\
4 & 5 & 6.6 \\
5 & 5 & 6.6 \\
\hline
\end{tabular}

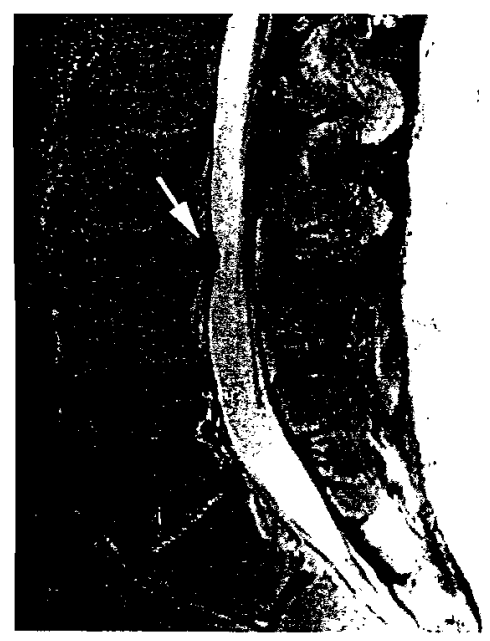

Fig. 1: Lumbar disc protrusion. L3 dise hemiation with the base of the hemiation wider than the distance away from the present disc. The protrusion is evident principally as a distortion of the cerebrospinal fluid-containing thecal sac on the T2-weighted images. 
Efficacy of dynamic muscular stabilization techniques - Suraj Kumar et al

Table 2: Homogeneity of variance between groups of pre outcome measures

\begin{tabular}{lllll}
\hline Outcome measures & \multicolumn{2}{c}{$\begin{array}{c}\text { Homogeneity of variance } \\
\text { P-value }\end{array}$} & F ratio & ANOVA \\
& Bartlett's statistics & P-value \\
\hline Pain & 2.67 & 0.6142 & 2.11 & 0.0880 \\
Walking & 7.11 & 0.1303 & 2.45 & 0.0536 \\
Stair climbing & 3.57 & 0.4670 & 0.56 & 0.6894 \\
Stand ups & 4.89 & 0.2993 & 2.01 & 0.1018 \\
BPC & 4.24 & 0.3751 & 1.34 & 0.2628 \\
APC & 8.99 & 0.0612 & 0.96 & 0.4329 \\
QOL & 5.97 & 0.2013 & 0.72 & 0.5823 \\
\hline
\end{tabular}

Table 3: Summary statistics (mean $\pm \mathrm{SE}$ ) of outcome measures

\begin{tabular}{lllllll}
\hline Variables & Periods & 1 disc & 2 disc & 3 disc & 4 disc & 5 disc \\
\hline Pain & Pre & $6.45 \pm 0.20$ & $6.32 \pm 0.24$ & $7.33 \pm 0.29^{12}$ & $6.80 \pm 0.37$ & $7.40 \pm 0.40$ \\
& Post & $1.37 \pm 0.12$ & $1.21 \pm 0.12$ & $1.22 \pm 0.22$ & $1.20 \pm 0.37$ & $2.60 \pm 0.24^{13}$ \\
Walking & Pre & $243.63 \pm 5.74$ & $232.16 \pm 8.54$ & $224.78 \pm 8.92$ & $221.60 \pm 6.68$ & $200.00 \pm 7.07^{1}$ \\
& Post & $286.71 \pm 5.67$ & $274.32 \pm 7.91$ & $258.00 \pm 7.90$ & $249.60 \pm 8.98$ & $223.60 \pm 7.33^{12}$ \\
Stair climbing & Pre & $24.97 \pm 0.89$ & $24.53 \pm 1.23$ & $23.56 \pm 1.45$ & $24.00 \pm 1.41$ & $21.60 \pm 1.36$ \\
& Post & $42.34 \pm 1.40$ & $41.00 \pm 1.74$ & $37.67 \pm 1.65$ & $34.80 \pm 1.93$ & $30.80 \pm 1.24^{12}$ \\
Stand ups & Pre & $17.24 \pm 0.77$ & $14.89 \pm 0.73$ & $13.56 \pm 1.09$ & $15.20 \pm 1.62$ & $14.80 \pm 2.48$ \\
& Post & $35.55 \pm 1.31$ & $35.42 \pm 1.75$ & $33.78 \pm 2.05$ & $33.40 \pm 1.50$ & $28.40 \pm 4.23$ \\
BPC & Pre & $19.87 \pm 0.92$ & $20.42 \pm 1.59$ & $16.89 \pm 1.60$ & $16.40 \pm 1.36$ & $16.20 \pm 2.01$ \\
& Post & $35.63 \pm 0.88$ & $40.68 \pm 1.32^{1}$ & $31.67 \pm 1.46^{2}$ & $30.80 \pm 2.29^{2}$ & $29.80 \pm 2.22^{5}$ \\
APC & Pre & $6.82 \pm 0.60$ & $7.95 \pm 0.82$ & $6.56 \pm 0.77$ & $9.20 \pm 0.86$ & $6.40 \pm 0.51$ \\
& Post & $17.55 \pm 0.72$ & $17.58 \pm 0.70$ & $15.33 \pm 0.94$ & $17.40 \pm 0.87$ & $12.80 \pm 0.86^{1}$ \\
QOL & Pre & $99.89 \pm 1.69$ & $100.63 \pm 1.54$ & $99.67 \pm 3.84$ & $98.00 \pm 3.22$ & $93.00 \pm 2.86$ \\
& Post & $61.18 \pm 1.07$ & $62.89 \pm 1.72$ & $67.67 \pm 3.44$ & $72.60 \pm 3.96^{\prime}$ & $72.80 \pm 3.28^{\prime}$ \\
\hline
\end{tabular}

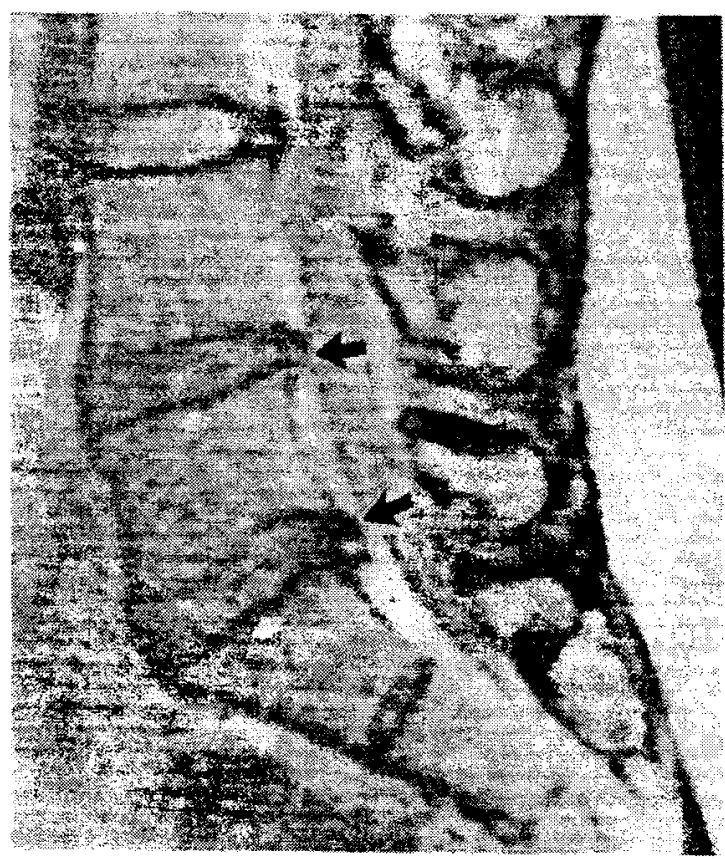

Fig. 2: On a T1-weighted saggital image, abnormal finding include posterior disc protrusion and decreased signal intensity in the nucleus pulposus at L4-L5 and L5-S1. 

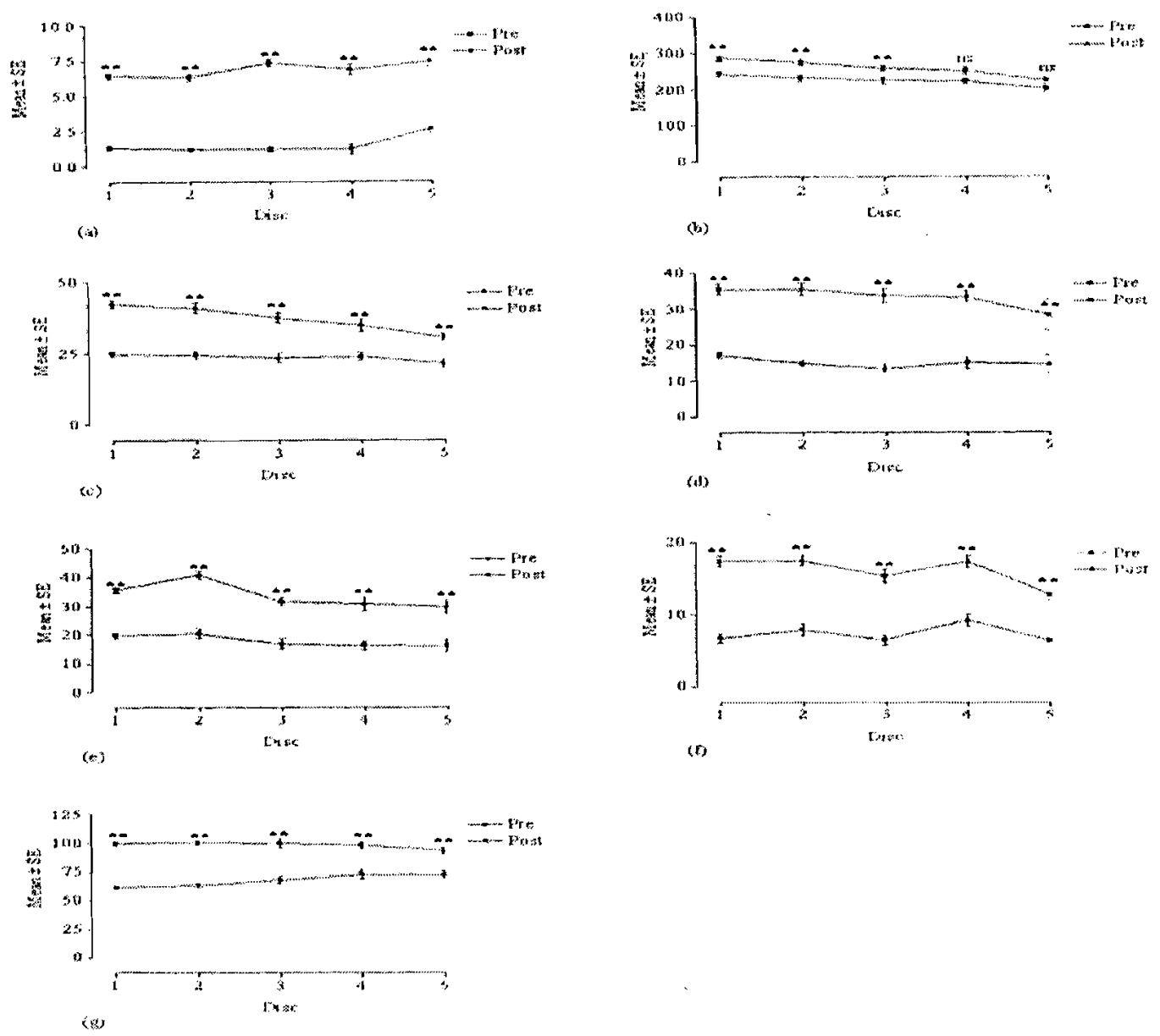

ns-P $>0.05, * *-\mathrm{P}<0.01$

Fig. 3: Represents pre and post mean level of pain (a), walking (b), stair climbing (c), stand ups (d), PBC (e), APC (f) and QoL (g) in different disc involved groups and compares the groups between the periods.

In the present study DMST was found to be effective in the management of low back pain even with multiple discs involvement. The hypothesis that the DMST treatment is equally effective in all groups was therefore rejected. The improvement due to DMST training in the 1 disc involvement group was significantly higher while compared to the group involving 2, 3, 4 and 5 discs. The mechanism by which DMST improved 1 disc may be due to restoration of muscle strength, balance, posture, position and coordination that otherwise would have been impaired in the presence of pain and functional disability.

Previous study showed that the functional status is impaired in the patients having LDD \& chronic low back pain, associated with degenerative changes on the MRI of the lumbo-sacral spine (Panagiotis et al, 2002). Our MRI finding also suggests more the disc involve, more the severity of pain and disability. Paul found no significant association between segmental distribution of symptoms and presence of anatomic impairment (Paul et al, 2000).

Previous physiotherapy programs have shown efficacy in patients with chronic low back pain (Furlan et al, 2001). The spinal physiotherapy program was concerned with the physiotherapy of muscles, and the progression contraction of these muscles into every day postures and positions, especially those associated with pain or functional disability. As a component of musculoskeletal physiotherapy, the spinal stabilization program is more effective than manually applied therapy or an education booklet in treating low back pain (Lucy et al, 2006).

Correct and timely rehabilitation is a vital component for the treatment of LBP patients. The goals of rehabilitation include restoring function, restoring pain free full range of motion, and achieving full muscle strength and endurance. This paper discusses the rehabilitation of LBP by the application of DMST with a special focus on the transverses abdominus (TA) and multifidi (MF) muscles. Lumbar stabilization exercises are aimed at sensorimotor reprogrammation of spine stabilizer muscles intended to improve their motor control skill and improve the response to compensate for weakness of the passive stabilization 
system. Our results can be generalized to LBP patients. Before implementing on general population it should be confirmed on more subjects, which is our future objective.

Clinical trials evaluating the efficacy of a variety of interventions for chronic non-specific low back pain indicate limited effectiveness of the most commonly applied interventions and approaches. The effects of treatment may be diluted by the application of a single intervention to a complex, heterogeneous group with diverse treatment needs (Wand and O'Connell, 2008). In our earlier study we have reported this may also be due to differences in genders (Kumar et al, 2009).

\section{CONCLUSION}

This study concludes that pain, walking, stair climbing and BPC improved maximally in the subject involving lesser number of discs whereas the quality of life improved more in the subjects with higher number of discs involved. In summary, the improvement was observed more on 1 (single) disc involved followed by multiple $(2,3,4$ and 5) discs involvement.

\section{ACKNOWLEDGEMENT}

Authors are thankful to Director, CDRI, for considering MPS Negi to analyze this as a collaborative work. We acknowledge Mr. Vishal and Mr. Lal Bahadur for their help during different phases of work. Authors want to acknowledge all the participants who participated in the study. Author (SK) acknowledges to ICMR, New Delhi for providing a Senior Research fellowship (letter no. 3/1/ 2/1/ADR/2007-NCD-I). This study has no conflict of interest.

\section{REFERENCES}

- Borenstein DG (1996). Chronic low back pain. Rheum Dis. Clin. North AM 22: 590-7.

- Furlan AD, Clarke J, Esmail R, Sinclair S, Irvin E (2001). A critical review of reviews on the treatment of chronic low back pain. Spine 26: E155-62.

- Hides JA, Jull GA, Richardson CA (2001). Long term effects of specific stabilizing exercises for first-episode low back pain. Spine 11, 243-248.

- Jensen MP, Chen C, Bruger AM (2002). Postsurgical pain outcome assessment. Pain 99, 101-109.

- Koes BW, Van Tulder MW, Thomas S (2006). Diagnosis and treatment of low back pain - Clinical review, $B M J 332$ :
$1430-4$.

- Kumar S, Negi MPS, Sharma VP (2008) b. Efficacy of dynamic muscular stabilization techniques (DMST) over conventional techniques in rehabilitation of chronic low back pain. Journal of sports conditioning and research 1, In Press.

- Kumar S, Negi MPS, Sharma VP, Shukla R (2008) a. The spinal column and abdominal pressure changes and their relationship with pain severity in patients with low back pain. Nigerian Journal of Medical Rehabilitation 13(21): 16.

- Suraj Kumar, Vijai P. Sharma, H K Tripathi, Mahendra P.S Negi, G.Venu Vendhan, "Efficacy of dynamic muscular stabilization techniques (DMST) over conventional techniques in patients with chronic low back pain". Indian Journal of Physiotherapy and Occupational Therapy 2009; 3(2): 47-53.

- Lucy JG, Ann PM (2006). A randomized controlled trial investigating the efficacy of musculoskeletal physiotherapy on chronic low back pain, Spine 3: 1083-1093.

- Martin P, Rose M, Nichols P, Russell P, Hughes I (1986). Physiotherapy exercises for low back pain process and clinical outcome. International Rehabilitation Medicine 8: 261-287.

- Panagiotis K. Anastassion D. Panos I. Elias L (2002). Corrective Analysis of Lateral Vertcbral Radiographic Variables and Medical Outcomes Study Short Form Health Servey: A Comparative Study in Asymptomatic Volunteers versus Patients with Low Back Pain. Journal of Spinal Disorder and Technique 15(5): 384-390.

- Paul BF, Steven MP, Paul S, Richard MW, Gary HM (2000). Association between patient report of symptoms and anatomic impairment visible on lumbar magnetic resonance imaging. Spine 25(7): 819-828.

- Richardson CA, Jull CA (1995). Muscle Control-Pain Control. What exercises would you prescribe? Man Ther 1: 2-10.

- Salo S, Paajaen H, and Alanen A (1995). Disc degeneration of pediatric patients in Lumbar MRI. Pediatric Radiology 25: $186-189$.

- Waddell G (1998). Functional capacity evaluation, The back pain revolution. New York, Churchill Livingstone, 41.

- Wand BM and O'Connell NE (2008). Chronic non-specific low back pain - sub-group or a single mechanism? BMC Musculoskeletal Disorder 9, 11 . 